\title{
Effect Of Perturbations In the Coriolis And Centrifugal Forces On The Libration Points In The R3BP When The Primaries Are Heterogeneous Spheroids With Three Layers
}

\author{
Jagadish Singh ${ }^{1}$, Benjamin Idenyi Salifu ${ }^{2}$ \\ ${ }^{1}$ Department of Mathematics, Faculty of Science, Ahmadu Bello University, Zaria, Nigeria. \\ ${ }^{2}$ Department of Mathematics, Faculty of Science, Ahmadu Bello University, Zaria, Nigeria. \\ Email: jgds2004@yahoo.com, salifubenjamin@gmail.com \\ DOI: 10.31364/SCIRJ/v8.i11.2020.P1120821 \\ http://dx.doi.org/10.31364/SCIRJ/v8.i11.2020.P1120821
}

\begin{abstract}
This study examines the combined effect of small perturbations in the Coriolis and centrifugal forces together with heterogeneity of two primaries with three layers of different densities on the positions and stability of an infinitesimal mass in the framework of the planar circular restricted three-body problem (R3BP). The existence of five libration points are observed, and they are seen to be affected by a small perturbation in the centrifugal force, and heterogeneity of the primaries. The stability of the collinear points is not influenced by the perturbations and heterogeneity and they remain unstable, while
\end{abstract} the triangular points are stable for $0<\mu<\mu_{c}$ and unstable for $\mu_{c} \leq \mu \leq \frac{1}{2}$, where $\mu_{C}$ is the critical mass value. It is also found that all perturbing factors except that of the Coriolis force have destabilizing behavior. Finally, a numerical exploration is also performed.

Keywords: Restricted three body; Libration points; Stability; Heterogeneous spheroid; Perturbations.

\section{Introduction}

The circular restricted three- body problem (CR3BP) describes the motion of an infinitesimal mass moving under the gravitational effects of two finite masses, called primaries, which move in circular orbits around their common center of mass. Wintner[1] showed that the stability of the triangular equilibrium points is due to the existence of the Coriolis terms in the equations of motion when these equations are recorded in the rotating coordinate system. Szebehely[2] noticed that CR3BP possesses five stationary solutions called Lagrangian points.Three are collinear with the primaries and the other two are in equilateral triangular configuration with the primaries. The three collinear points $L_{1,2,3}$ are unstable, while the triangular points $L_{4,5}$ are stable for the mass ratio 
$\mu=\frac{m_{2}}{m_{1}+m_{2}}<0.03852 \ldots, m_{1} \geq m_{2}$ being the masses of the primaries. Chernikov [3] studied Lagrangian points in the photogravitational restricted three- body problem. Sharma[4] examined the linear stability of triangular libration points of the restricted three body problem when the more massive primary is a source of radiation and an oblate spheroid as well. He found that the eccentricity of the conditional retrograde elliptic periodic orbits around the triangular points at the critical mass increases with the increase in the oblateness coefficient and the radiation force and becomes unity when $\mu_{c}$ is zero. Simons etal.[5] gave a complete solution of the restricted three-body problem. They discussed the existence and linear stability of the equilibrium points for all values of radiation pressures of both luminous bodies and all values of mass ratios. Ragos and Zagouras[6] found two families of periodic solutions about the 'out of plane' equilibrium points in the photogravitational restricted three body problem. Shaboury[7] gave a possibility of nine libration points for small values of oblateness in the photogravitational restricted three-body problem when the infinitesimal mass is of an axisymmetric body and one of the finite masses be a spherical luminous body while the other be an axisymmetric non-luminous body. Khanna and Bhatnagar[8] discussed the stationary solutions of the planar R3BP when the smaller primary is a triaxial rigid body with one of the axes as the axis of symmetery and its equatorial plane coinciding with the plane of motion. The bigger primary is taken as an oblate spheroid and its equatorial plane is also coinciding with the plane of motion. They found that the collinear points are unstable, while the triangular points are stable for the mass parameter $0<\mu<\mu_{\text {crit }}$ and the triangular points have long or short periodic elliptical orbits in the same range of $\mu_{c r i t}$.

Idrisi[9] have studied the R3BP by taking one of the primaries as an ellipsoid. They used the elliptic integrals to write the equations of motion and investigated the collinear and non collinear libration points and their stability in linear sense. They observed that there exist five collinear libration points and the non collinear libration points lying on the arc of a unit circle, whose centre is the bigger primary. They further observed that the libration points either collinear or non-collinear are all unstable.

Singh and Taura[10] examined the stability of triangular libration points in the photogravitational R3BP with Oblateness and potential from a belt. Md Sanam Suraj etal.[11] investigated the photo-gravitational R3BP when the primaries are heterogeneous spheroid with three layers. Singh and Nakone[12] studied the effect of perturbations in the Coriolis and centrifugal forces on the stability of $L_{4}$ in the relativistic $\mathrm{R} 3 \mathrm{BP}$ and found that libration points are slightly affected by a small change in the centrifugal force. Singh and Taura[13] discussed collinear libration points in the photogravitational CR3BP with zonal harmonics and the Potential from a belt and found that the existence of new collinear points can be utilized as stations for artificial satellites.

The focus of this paper is to study the combined effect of small perturbations in the Coriolis and centrifugal forces together with heterogeneity of the primaries on locations and stability of libration points.

$$
\text { www.scirj.org }
$$

(C) 2020, Scientific Research Journal 
The paper is structured as follows:

\section{Equations of motion}

Let $m_{1}$ and $m_{2}$ be the masses of the primary and secondary finite bodies respectively, and $m$ be the mass of the third body. Using dimensionless variables, the equations of motion of the third body in a barycentric rotating coordinate system, can be written as

$\ddot{x}-2 \alpha n \dot{y}=\Omega_{x}$

$\ddot{y}+2 \alpha n \dot{x}=\Omega_{y}$

Where

$\Omega=\frac{n^{2} \beta\left(x^{2}+y^{2}\right)}{2}+\frac{1-\mu}{r_{1}}+\frac{k_{1}}{2 r_{1}^{3}}+\frac{\mu}{r_{2}}+\frac{k_{2}}{2 r_{2}^{3}}$

$n^{2}=1+\frac{3}{2} k_{3}$

$r_{1}^{2}=(x-\mu)^{2}+y^{2}$

$r_{2}^{2}=(x-\mu+1)^{2}+y^{2}$

$\alpha=1+\varepsilon$ and $\beta=1+\varepsilon^{\prime}$,

where $\varepsilon$ and $\varepsilon^{\prime}$ are very small perturbations in the Coriolis and centrifugal forces respectively, $n^{2}$ is the mean motion of the primaries,

$r_{1}$ is the distance of the infinitesimal mass from the bigger primary $m_{1}$,

$r_{2}$ is the distance of the infinitesimal mass from the smaller primary $m_{2}$,

$\mu$ is the critical mass parameter,

$k_{1}, k_{2}$ and $k_{3}$ are the heterogeneous factors of the primaries.

\section{Location of equilibrium points:}

The equilibrium points represent stationary solutions of the equations $\Omega_{x}=0, \Omega_{y}=0$

3.1 Location of triangular points:

Triangular points are the solutions of the equations $\Omega_{x}=0, \Omega_{y}=0$ and $y \neq 0$, this yields 


$$
\begin{aligned}
& \beta\left(1+\frac{3 k_{3}}{2}\right) x-\frac{(x-\mu)(1-\mu)}{r_{1}^{3}}-\frac{3 k_{1}(x-\mu)}{2 r_{1}^{5}}-\frac{\mu(x-\mu+1)}{r_{2}^{3}}-\frac{3 k_{2}(x-\mu+1)}{2 r_{2}^{5}}=0 \\
& \beta\left(1+\frac{3 k_{3}}{2}\right) y-\frac{y(1-\mu)}{r_{1}^{3}}-\frac{3 k_{1} y}{2 r_{1}^{5}}-\frac{\mu y}{r_{2}^{3}}-\frac{3 k_{2} y}{2 r_{2}^{5}}=0
\end{aligned}
$$

Since $y \neq 0$, we have

$\beta\left(1+\frac{3 k_{3}}{2}\right)-\frac{(1-\mu)}{r_{1}^{3}}-\frac{3 k_{1}}{2 r_{1}^{5}}-\frac{\mu}{r_{2}^{3}}-\frac{3 k_{2}}{2 r_{2}^{5}}=0$

If the bodies are not heterogeneous spheroids then $k_{1}=k_{2}=k_{3}=0$ and (5) becomes

$$
\begin{aligned}
& \beta x-\frac{(x-\mu)(1-\mu)}{r_{1}^{3}}-\frac{\mu(x-\mu+1)}{r_{2}^{3}}=0 \\
& \beta y-\frac{y(1-\mu)}{r_{1}^{3}}-\frac{\mu y}{r_{2}^{3}}=0 \quad y \neq 0
\end{aligned}
$$

Rewriting (7) as

$$
\left[\beta-\frac{(1-\mu)}{r_{1}^{3}}-\frac{\mu}{r_{2}^{3}}\right] x+(1-\mu) \mu\left(\frac{1}{r_{1}^{3}}-\frac{1}{r_{2}^{3}}\right)=0
$$

And rewriting (8) we have

$$
\left[\beta-\frac{(1-\mu)}{r_{1}^{3}}-\frac{\mu}{r_{2}^{3}}\right]=0
$$

Making use of (10) in (9) we have

$$
\frac{1}{r_{1}^{3}}-\frac{1}{r_{2}^{3}}=0
$$

A substitution of (11) in (10) provides

$$
r_{1}=\frac{1}{\beta^{\frac{1}{3}}} \text { and } r_{2}=\frac{1}{\beta^{\frac{1}{3}}} \text {. }
$$

If the bodies are heterogeneous spheroids, then the values of $r_{1}$ and $r_{2}$ will change slightly i.e 
$r_{1}=\left(\frac{1}{\beta}\right)^{\frac{1}{3}}+\epsilon_{1}$

$r_{2}=\left(\frac{1}{\beta}\right)^{\frac{1}{3}}+\epsilon_{2}$

where $\epsilon_{1}$ and $\epsilon_{2}$ and are very small.

The exact coordinates of the triangular points can be obtained from (4) as

$$
\begin{aligned}
& x=\mu-\frac{1}{2}+\frac{r_{2}^{2}-r_{1}^{2}}{2} \\
& y= \pm\left[\frac{r_{1}^{2}+r_{2}^{2}}{2}-\frac{1}{4}-\frac{\left(r_{2}^{2}-r_{1}^{2}\right)}{4}\right]^{\frac{1}{2}}
\end{aligned}
$$

Putting the values of $r_{1}$ and $r_{2}$ from (12) and (13) in (14) and (15) and ignoring terms of second and higher powers of $\epsilon_{1}$ and $\epsilon_{2}$, we have

$$
x=\mu-\frac{1}{2}+\beta^{-\frac{1}{3}}\left(\epsilon_{2}-\epsilon_{1}\right)
$$

$y^{2}=\beta^{-\frac{2}{3}}+\beta^{-\frac{1}{3}}\left(\epsilon_{2}+\epsilon_{1}\right)-\frac{1}{4}-\frac{2 \beta^{-\frac{1}{3}}}{4}\left(\epsilon_{2}-\epsilon_{1}\right)$

Substituting the values of $r_{1}, r_{2} x$ and $y$ from (12) and (13), (16) and (17) in (5) and (6) and then solving them using $\beta=1+\varepsilon$ and neglecting terms of very small quantities, we have

$$
\begin{aligned}
& (2 \mu-2) \varepsilon_{1}+2 \mu \varepsilon_{2}=-k_{1}+k_{2}+(1-2 \mu) k_{3} \\
& (2-2 \mu) \varepsilon_{1}+2 \mu \varepsilon_{2}=k_{1}+k_{2}-k_{3}
\end{aligned}
$$

Solving (18) and (19) simultaneously we have

$$
\varepsilon_{1}=\frac{k_{1}}{2(1-\mu)}-\frac{k_{3}}{2} \text { and } \varepsilon_{2}=\frac{k_{2}}{2 \mu}-\frac{k_{3}}{2}
$$

Putting these values in (16) and (17), we get

$$
x=\mu-\frac{1}{2}+\frac{1}{2}\left(\frac{k_{2}}{\mu}-\frac{k_{1}}{1-\mu}\right)
$$


$y= \pm \frac{\sqrt{3}}{2}\left[1-\frac{4 \varepsilon}{9}+\frac{1}{3}\left(\frac{k_{1}}{1-\mu}+\frac{k_{2}}{\mu}\right)-\frac{2 k_{3}}{3}\right]$

Since $r_{1} \neq r_{2} \neq 1$,each of the two points represented by (20) and (21) form scalene triangles with the primaries. They are denoted by $L_{4}(x, y)$ and $L_{5}(x,-y)$ and are known as the triangular points. The positions of these points depend on a small perturbation $\varepsilon$ in the centrifugal force and heterogeneity of the primaries. The absence of $\varepsilon$ shows that the triangular points are not affected by the perturbation given to the Coriolis force.

\subsection{Location of collinear points}

The solutions of $\Omega_{x}=0, \Omega_{y}=0, y=0 \quad$ yield the collinear points. The collinear points lie on the line joining the primaries. Putting $y=0$ in (5) we obtain

$n^{2} \beta x-\frac{(1-\mu)(x-\mu)}{|x-\mu|^{3}}-\frac{3 k_{1}(x-\mu)}{2|x-\mu|^{5}}-\frac{\mu(x-\mu+1)}{|x-\mu+1|^{3}}-\frac{3 k_{2}(x-\mu+1)}{2|x-\mu+1|^{5}}=0$

To locate the collinear points, we divide the orbital plane Oxy into three parts with respect to the primaries

Case $1:-\infty<x<\mu-1$

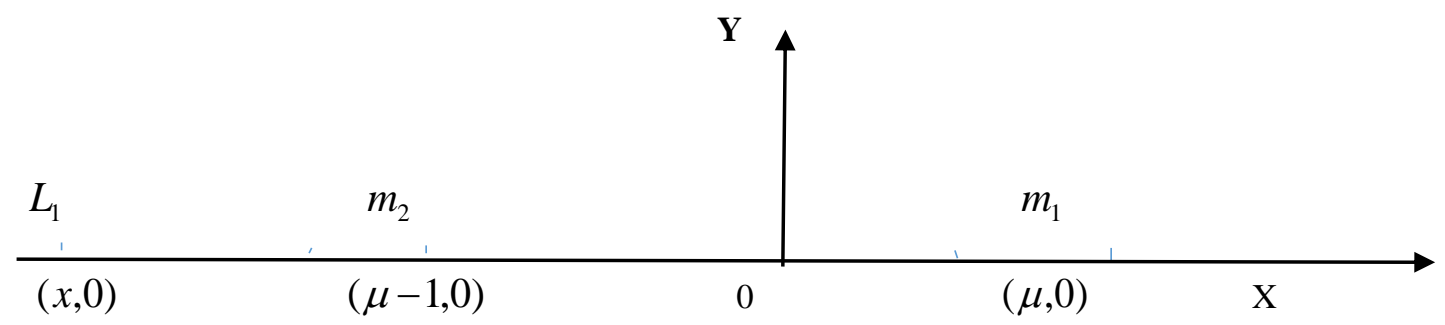

Let $x=\mu-1-\tau_{1}$ be the abscissa of the point lying in the part $-\infty<x<\mu-1$ at a distance $\tau_{1}$ from the smaller primary.

Substituting $x=\mu-1-\tau_{1}$ in (22) we have

$$
\begin{aligned}
& -2 n^{2} \beta \tau_{1}^{9}-2 n^{2} \beta[4+(1-\mu)] \tau_{1}^{8}-2\left[n^{2} \beta(6+4(1-\mu))\right] \tau_{1}^{7}-2\left[n^{2} \beta(4+6(1-\mu))-(1-\mu)-\mu\right] \tau_{1}^{6} \\
& -2\left[n^{2} \beta(1+4(1-\mu)-2(1-\mu)-4 \mu)\right] \tau_{1}^{5}-2\left[n^{2} \beta(1-\mu)-(1-\mu)-\frac{3 k_{1}}{2}-6 \mu-\frac{3 k_{2}}{2}\right] \tau_{1}^{4} \\
& +2\left[\left(4 \mu+6 k_{2}\right)\right] \tau_{1}^{3}+2\left[\mu+9 k_{2}\right] \tau_{1}^{2}+12 k_{2} \tau_{1}+3 k_{2}=0
\end{aligned}
$$


This is an algebraic polynomial of degree nine in $\tau_{1}$. Since there is only one change of sign, there exists only one real root.

Case 2: $\mu-1<x<\mu$

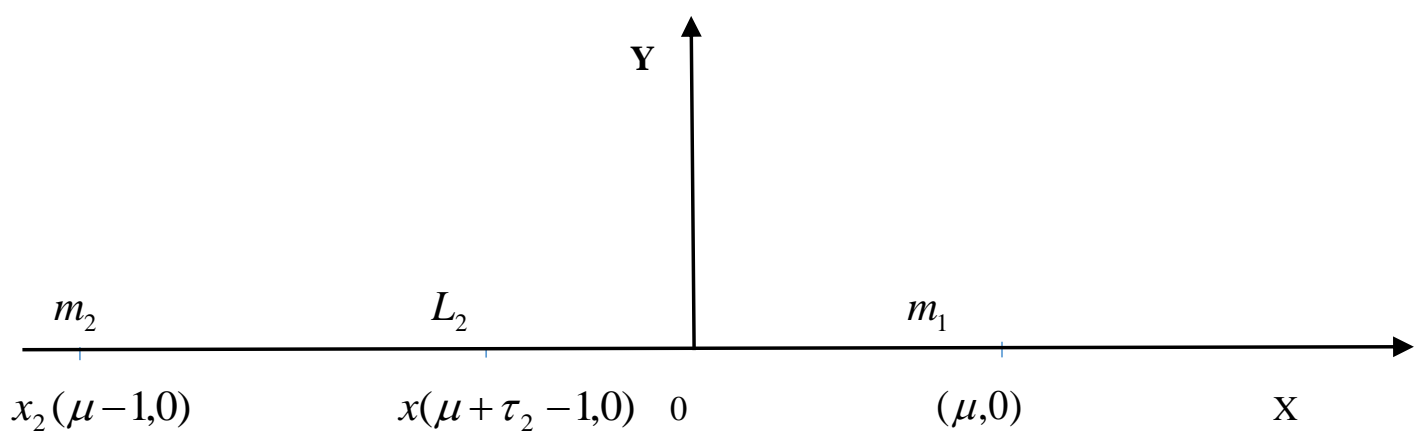

Let $x=\mu-1+\tau_{2}$ be the abscissa of the point lying in the part $\mu-1<x<\mu$ at a distance $\tau_{2}$ from the smaller primary , putting $x=\mu-1+\tau_{2}$ in (22), we obtain

$$
\begin{aligned}
& 2 n^{2} \beta \tau_{2}^{9}-2 n^{2} \beta[4+(1-\mu)] \tau_{2}^{8}+2 n^{2} \beta[6+4(1-\mu)] \tau_{2}^{7}-2\left[n^{2} \beta(4+6(1-\mu)-(1-\mu)+\mu)\right] \tau_{2}^{6} \\
& +2\left[n^{2} \beta(1+4(1-\mu)-2(1-\mu)+4 \mu)\right] \tau_{2}^{5}-2\left[n^{2} \beta(1-\mu)-(1-\mu)-\frac{3 k_{1}}{2}+6 \mu+\frac{3 k_{2}}{2}\right] \tau_{2}^{4} \\
& +2\left[4 \mu+6 k_{2}\right] \tau_{2}^{3}-2\left[\mu-9 k_{2}\right] \tau_{2}^{2}+12 k_{2} \tau_{2}-3 k_{2}=0
\end{aligned}
$$

This is a ninth degree polynomial with only one change of sign. Hence there exist only one real root.

Case3: $\mu<x<\infty$

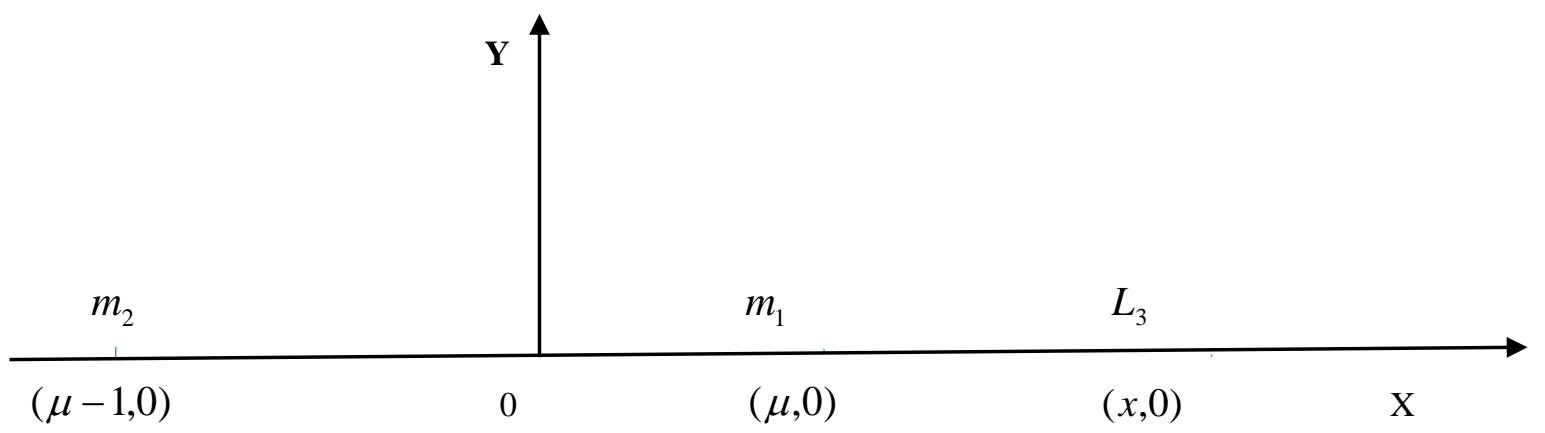

Let $x=\mu+\tau_{3}$ be the abscissa of the point lying in the part $\mu<x<\infty$ at a distance $\tau_{3}$ from the bigger primary. Then substituting this value of $x$ in (22) we get

$$
2 n^{2} \beta \tau_{3}^{9}+2 n^{2} \beta[4+\mu] \tau_{3}^{8}+4 n^{2} \beta[3+2 \mu] \tau_{3}^{7}+2\left[2 n^{2} \beta(2+3 \mu)-(1-\mu)-\mu\right] \tau_{3}^{6}
$$




$$
\begin{aligned}
& +2\left[n^{2} \beta(1+4 \mu)-4(1-\mu)-2 \mu\right] \tau_{3}^{5}+2\left[n^{2} \beta \mu-6(1-\mu)-\frac{3 k_{1}}{2}-\left(\mu+\frac{3 k_{2}}{2}\right)\right] \tau_{3}^{4} \\
& -4\left[2(1-\mu)+3 k_{1}\right] \tau_{3}^{3}-2\left[(1-\mu)+9 k_{1}\right] \tau_{3}^{2}-12 k_{1} \tau_{3}-3 k_{1}=0
\end{aligned}
$$

This is a ninth degree polynomial with only one change of sign. Hence there exists only one real root.

We shall locate the collinear points numerically in section 5 by solving equations (23), (24) and (25).

\subsection{Stability of libration points:}

The libration point is stable if all the roots of the characteristic equation are either negative real numbers or distinct pure imaginary numbers or real parts of the complex roots are negative.

we denote any equilibrium point and its position as $L\left(x_{0}, y_{0}\right)$. To test stability,consider a small displacement $(\xi, \eta)$ from the point such that $x=x_{0}+\xi$ and $y=y_{0}+\eta$.Substituting these values in (1), we obtain the variational equations

$$
\begin{aligned}
& \ddot{\xi}-2 n \alpha \dot{\eta}=\Omega_{x x}^{0}+\Omega_{x y}^{0} \eta \\
& \ddot{\eta}+2 n \alpha \dot{\xi}=\Omega_{x y}^{0}+\Omega_{y y}^{0} \eta
\end{aligned}
$$

where the superscript 0 indicates that the partial derivatives denoted by subscripts are evaluated at the equilibrium point $\left(x_{0}, y_{0}\right)$.

Their characteristic equation is

$$
\lambda^{4}+\left(4 n^{2} \alpha^{2}-\Omega_{x x}^{0}-\Omega_{y y}^{0}\right) \lambda^{2}+\Omega_{x x}^{0} \Omega_{y y}^{0}-\left(\Omega_{x y}^{0}\right)^{2}=0
$$

\subsection{Stability of triangular points}

For the triangular points we have

$$
\begin{aligned}
& \Omega_{x x}^{0}=\frac{3}{4}+\frac{5 \varepsilon^{\prime}}{4}+\frac{3 k_{1}}{8}+\frac{9 k_{1}}{8(1-\mu)}-\frac{21 \mu k_{1}}{8(1-\mu)}+3 k_{2}-\frac{3 k_{2}}{2 u}+\frac{15 k_{3}}{8} \\
& \Omega_{y y}^{0}=\frac{9}{4}+\frac{7 \varepsilon^{\prime}}{4}+\frac{33 k_{1}}{8}-\frac{21 k_{1}}{8(1-\mu)}+\frac{33 \mu k_{1}}{8(1-\mu)}+\frac{3 k_{2}}{2 \mu}+\frac{21 k_{3}}{8} \\
& \Omega_{x y}^{0}=\frac{-3 \sqrt{3}}{4}+\frac{3 \mu \sqrt{3}}{2}-\frac{11 \sqrt{3} \varepsilon^{\prime}}{12}+\frac{11 \mu \sqrt{3} \varepsilon^{\prime}}{6}-\frac{11 \mu \sqrt{3} k_{1}}{8(1-\mu)}+\frac{7 \sqrt{3} k_{1}}{8(1-\mu)}-\frac{15 \sqrt{3} k_{1}}{8}+\frac{\sqrt{3} k_{2}}{2}+\frac{\sqrt{3} k_{2}}{2 \mu}-\frac{11 \sqrt{3} k_{3}}{8}
\end{aligned}
$$

Replacing $\lambda^{2}$ by $v$ the characteristic equation (26) becomes

$$
v^{2}+b v+c=0
$$


The roots of (30) are $v_{1,2}=\frac{-b \pm \sqrt{\Delta}}{2}$ where $\Delta=b^{2}-4 c$ is the discriminant,

$b=1+8 \varepsilon-3 \varepsilon^{\prime}-3 k_{1}-3 k_{2}+\frac{3 k_{3}}{2}$ and

$c=\frac{27 \mu}{4}-\frac{27 \mu^{2}}{4}+\frac{33 \mu \varepsilon}{2}-\frac{33 \mu^{2} \varepsilon}{2}+\frac{9 k_{1} \mu}{2}+\frac{9 k_{2}}{2}-\frac{9 \mu k_{2}}{2}+\frac{99 \mu k_{3}}{4}-\frac{99 \mu^{2} k_{3}}{4}$

The discriminant can be expressed as

$$
\Delta=f(\mu)=\psi_{1} \mu^{2}+\psi_{2} \mu+\psi_{3}
$$

where $\psi_{1}=27+66 \varepsilon^{\prime}+99 k_{3}, \psi_{2}=18 k_{2}-99 k_{3}-18 k_{1}-66 \varepsilon^{\prime}-27$,

$\psi_{3}=1+16 \varepsilon-6 \varepsilon^{\prime}-6 k_{1}-24 k_{2}+3 k_{3}$

Now $f(0)=1+16 \varepsilon-6 \varepsilon^{\prime}-6 k_{1}-24 k_{2}+3 k_{3}>0$

$f\left(\frac{1}{2}\right)=-\frac{23}{4}+16 \varepsilon-\frac{21 \varepsilon^{\prime}}{2}-15 k_{1}-15 k_{2}-\frac{873 k_{3}}{4}<0$

Hence, there is only one value for $\mu$ say $\mu_{c}$ in the interval $\left(0, \frac{1}{2}\right)$ for which $\Delta$ vanishes. Now we consider the three regions of the value of $\mu$ separately.

(i) when $0<\mu<\mu_{c}, \Delta>0$; the values of $v$ are negative and therefore all the four characteristic roots are distinct pure imaginary numbers. Hence triangular points are stable.

(ii) when $\mu_{c} \leq \mu \leq \frac{1}{2}, \Delta<0$; the real parts of two of the characteristic roots are positive. Therefore the triangular points are unstable.

(iii) when $\mu=\mu_{c}, \Delta=0$ :the values of $v$ are same, hence triangular points are unstable. Thus for $0<\mu<\mu_{c}$ we have stability and for $\mu_{c} \leq \mu \leq \frac{1}{2}$ we have instability.

The solution of the quadratic equation $\Delta=0$ for $\mu$ gives the critical mass ratio of the mass parameter. That is $\mu_{c}=-\frac{\psi_{2}}{2 \psi_{1}} \pm \frac{\left(\psi_{2}^{2}-4 \psi_{1} \psi_{3}\right)^{\frac{1}{2}}}{2 \psi_{1}}$ i.e 
$\mu_{c}=\mu_{0}+\mu_{1}+\mu_{2}+\mu_{3}+\mu_{p}$

with

$\mu_{0}=\frac{1}{2}\left(1-\frac{\sqrt{69}}{9}\right)$

$\mu_{1}=\frac{1}{3}\left(1-\frac{15}{\sqrt{69}}\right) k_{1}$

$\mu_{2}=-\frac{1}{3}\left(1-\frac{15}{\sqrt{69}}\right) k_{2}$

$\mu_{3}=-\frac{2}{9 \sqrt{69}} k_{3}$

$\mu_{p}=\frac{4\left(36 \varepsilon-19 \varepsilon^{\prime}\right)}{27 \sqrt{69}}$

Here $\mu_{0}$ represents Routh's critical mass ratio; and $\mu_{1}, \mu_{2}$ and $\mu_{3}$ are effects arising from the three layers of different densities of the primaries. $\mu_{p}$ appears due to the effect of small perturbations in the Coriolis and centrifugal forces. In the absence of these heterogeneous factors, $\mu_{c}$ reduces to $\mu_{0}+\frac{4\left(36 \varepsilon-19 \varepsilon^{\prime}\right)}{27 \sqrt{69}}$ which agrees with the result of Bhatnagar and Hallan (1978).

\subsection{Stability of collinear points}

Stability of $L_{1}:-\infty<x_{0}<\mu-1$

In this case $y_{0}=0, x_{0}=\mu-1-\tau_{1}$. The partial derivative computed at the collinear point $\left(x_{0}, 0\right)$ are given by

$\Omega_{x x}^{0}=n^{2} \beta+\frac{2(1-\mu)}{\left|x_{0}-\mu\right|^{3}}+\frac{6 k_{1}}{\left|x_{0}-\mu\right|^{3}}+\frac{2 \mu}{\left|x_{0}-\mu+1\right|^{3}}+\frac{6 k_{2}}{\left|x_{0}-\mu+1\right|^{5}}$

$\Omega_{y y}^{0}=n^{2} \beta-\frac{(1-\mu)}{\left|x_{0}-\mu\right|^{3}}-\frac{3 k_{1}}{2\left|x_{0}-\mu\right|^{5}}-\frac{\mu}{\left|x_{0}-\mu+1\right|^{3}}-\frac{3 k_{2}}{2\left|x_{0}-\mu+1\right|^{5}}$

$\Omega_{x y}^{0}=\Omega_{y x}^{0}=0, \Omega_{x x}^{0}>0, \Omega_{y y}^{0}<0$

Stability of $L_{2}: \mu-1<x_{0}<\mu$

In this case $x_{0}=\mu-1+\tau_{2}, y_{0}=0$.The partial derivatives computed at the collinear point $\left(x_{0}, 0\right)$ are 


$$
\begin{aligned}
& \Omega_{x x}^{0}=n^{2} \beta+\frac{2(1-\mu)}{\left|x_{0}-\mu\right|^{3}}+\frac{6 k_{1}}{\left|x_{0}-\mu\right|^{5}}+\frac{2 \mu}{\left|x_{0}-\mu+1\right|^{3}}+\frac{6 k_{2}}{\left|x_{0}-\mu+1\right|^{5}} \\
& \Omega_{y y}^{0}=n^{2} \beta-\frac{(1-\mu)}{\left|x_{0}-\mu\right|^{3}}-\frac{3 k_{1}}{2\left|x_{0}-\mu\right|^{5}}-\frac{\mu}{\left|x_{0}-\mu+1\right|^{3}}-\frac{3 k_{2}}{2\left|x_{0}-\mu+1\right|^{5}} \\
& \Omega_{x y}^{0}=\Omega_{y x}^{0}=0, \Omega_{x x}^{0}>0, \Omega_{y y}^{0}<0
\end{aligned}
$$

Stability of $L_{3}: \mu<x_{0}<\infty$

In this case $y_{0}=0, x_{0}=\mu+\tau_{3}$. The partial derivative computed at the collinear point $\left(x_{0}, 0\right)$ are

$$
\begin{aligned}
& \Omega_{x x}^{0}=n^{2} \beta+\frac{2(1-\mu)}{\left|x_{0}-\mu\right|^{3}}+\frac{6 k_{1}}{\left|x_{0}-\mu\right|^{5}}+\frac{2 \mu}{\left|x_{0}-\mu+1\right|^{3}}+\frac{6 k_{2}}{\left|x_{0}-\mu+1\right|^{5}} \\
& \Omega_{y y}^{0}=n^{2} \beta-\frac{(1-\mu)}{\left|x_{0}-\mu\right|^{3}}-\frac{3 k_{1}}{2\left|x_{0}-\mu\right|^{5}}-\frac{\mu}{\left|x_{0}-\mu+1\right|^{3}}-\frac{3 k_{2}}{2\left|x_{0}-\mu+1\right|^{5}}
\end{aligned}
$$

$\Omega_{x y}^{0}=\Omega_{y x}^{0}=0, \Omega_{x x}^{0}>0, \Omega_{y y}^{0}<0$. Since for each case, $\Omega_{x y}^{0}=\Omega_{y x}^{0}=0$

The characteristic roots of (26)can be expressed as

$\lambda_{1,2}= \pm \frac{\left[-4 n^{2} \alpha^{2}+\Omega_{x x}^{0}+\Omega_{y y}^{0}+\sqrt{16 n^{4} \alpha^{4}-8 n^{2} \alpha^{2} \Omega_{x x}^{0}+\left(\Omega_{x x}^{0}\right)^{2}-8 n^{2} \alpha^{2} \Omega_{y y}^{0}+\left(\Omega_{y y}^{0}\right)^{2}-2 \Omega_{x x}^{0} \Omega_{y y}^{0}}\right]^{\frac{1}{2}}}{\sqrt{2}}$

$\lambda_{3,4}= \pm \frac{\left[-4 n^{2} \alpha^{2}+\Omega_{x x}^{0}+\Omega_{y y}^{0}-\sqrt{16 n^{4} \alpha^{4}-8 n^{2} \alpha^{2} \Omega_{x x}^{0}+\left(\Omega_{x x}^{0}\right)^{2}-8 n^{2} \alpha^{2} \Omega_{y y}^{0}+\left(\Omega_{y y}^{0}\right)^{2}-2 \Omega_{x x}^{0} \Omega_{y y}^{0}}\right]^{\frac{1}{2}}}{\sqrt{2}}$

Thus, at all the three collinear points, we have $\Omega_{x y}^{0}=\Omega_{y x}^{0}=0, \Omega_{x x}^{0}>0, \Omega_{y y}^{0}<0$ so that $\Omega_{x x}^{0} \Omega_{y y}^{0}<0$. this implies that the discriminant of the characteristic equation (26) is positive and its four roots can be written as $\lambda_{1}=s, \lambda_{2}=-s, \lambda_{3}=i s_{1}$, $\lambda_{4}=-i s_{1}$, where $s$ and $s_{1}$ are real. Therefore, the collinear points are unstable. 


\section{Numerical exploration}

we wish to apply the results obtained in sections 3 and 4 to a double star HD211415 which is visible to the naked eye $: \mu=0.3814$,

$k_{1}=1.58302 \times 10^{-7} ; k_{2}=9.83933 \times 10^{-18} ; k_{3}=3.13153 \times 10^{-8}$

Table1 Location of triangular points:

\begin{tabular}{lrl}
\hline$\varepsilon^{\prime}$ & $\boldsymbol{x}$ & $\boldsymbol{1}$ \\
\hline 0.00 & -0.1186 & 0.866025 \\
0.01 & -0.1186 & 0.862176 \\
0.06 & -0.1186 & 0.842931 \\
0.11 & -0.1186 & 0.804441 \\
0.21 & -0.1186 & 0.785196 \\
0.26 & -0.1186 & 0.765951 \\
0.31 & -0.1186 & 0.746706 \\
\hline
\end{tabular}

It shows the effect of a perturbation given to the centrifugal force. 


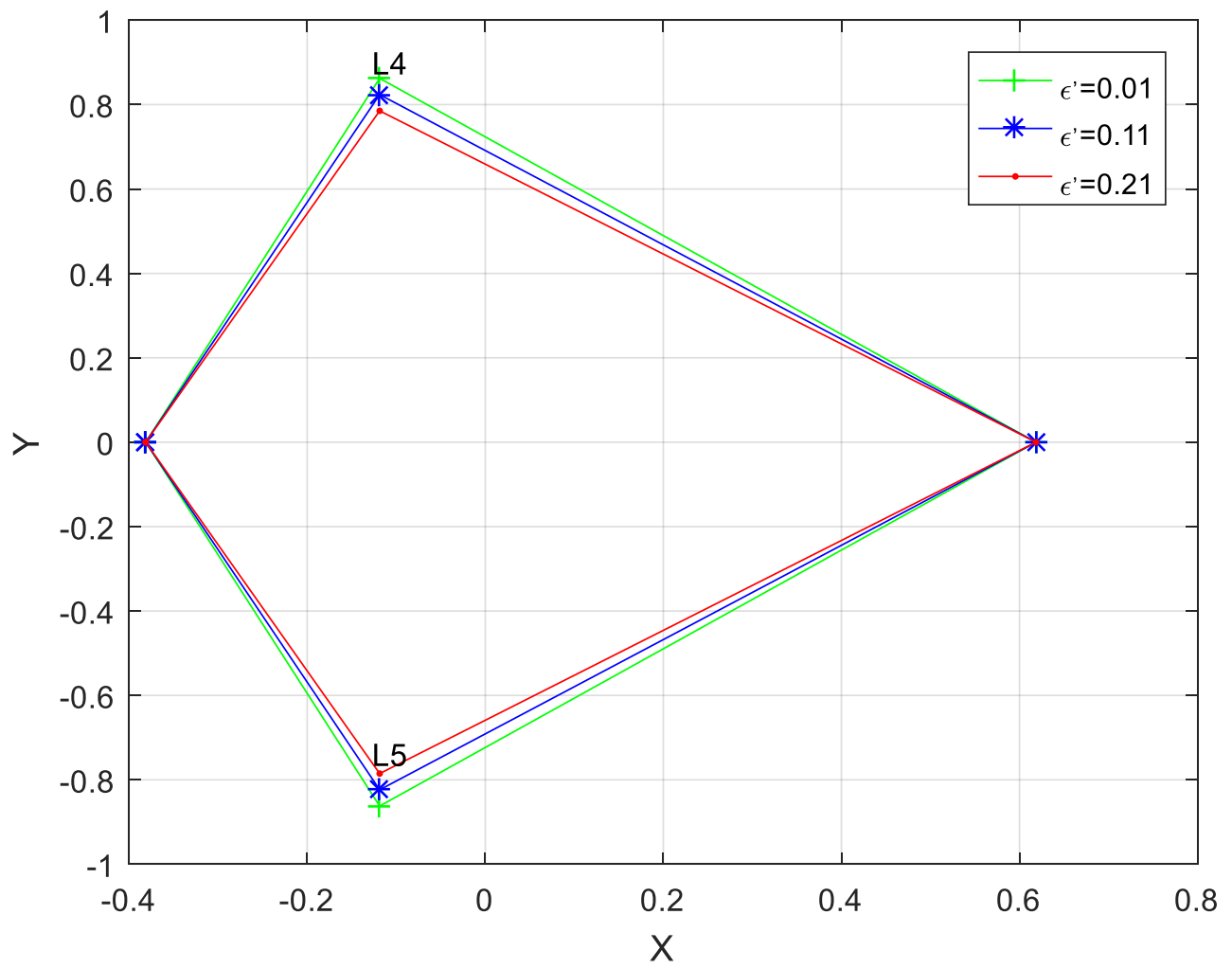

Figure 1 shows that increasing the perturbation in the centrifugal force brings triangular points closer to the $\mathrm{x}$-axis. Triangular points are not affected by the perturbation in the Coriolis force.

Table2 Location of collinear points:

\begin{tabular}{llll}
\hline$\varepsilon^{\prime}$ & $\boldsymbol{L}_{\mathbf{1}}$ & $\boldsymbol{L}_{2}$ & $\boldsymbol{L}_{3}$ \\
\hline 0.0 & -1.23623 & -0.168178 & 0.806425 \\
0.1 & -1.21074 & -0.167182 & 0.806425 \\
0.2 & -1.18834 & -0.166198 & 0.748024 \\
0.4 & -1.15065 & -0.164261 & 0.619602 \\
0.6 & -1.11994 & -0.162366 & 0.558745 \\
0.8 & -1.09428 & -0.160512 & 0.510383 \\
0.9 & -1.08292 & -0.159600 & 0.489858 \\
\hline
\end{tabular}

The table shows that an increase in the perturbation of the centrifugal force brings the collinear points towards the primaries. 


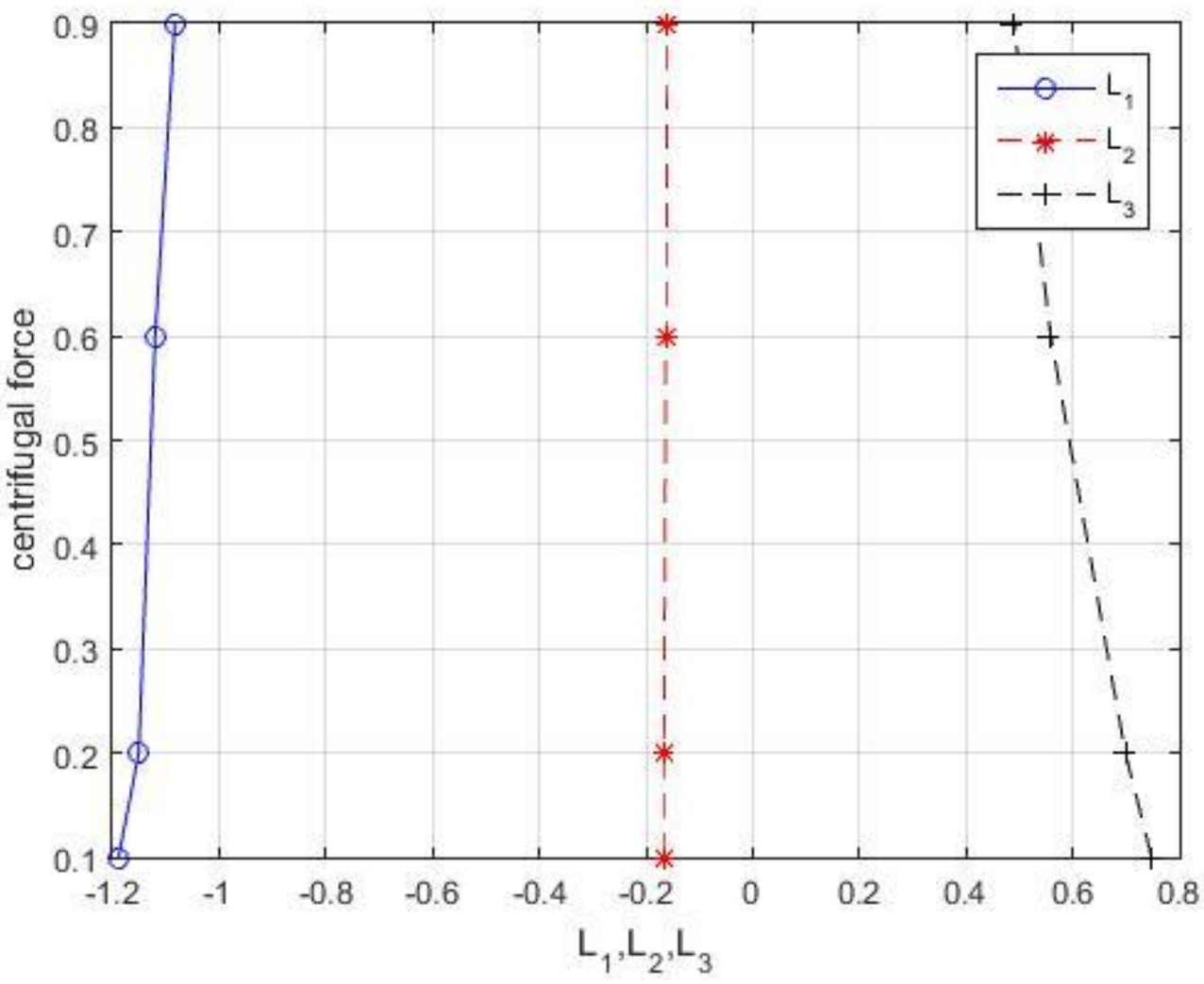

Figure2 shows that points $L_{1}, L_{2}, L_{3}$ remain collinear and lie on the x-axis.

\section{Stability of triangular points:}

Table3: Effect of a perturbation in the centrifugal force with heterogeneous factors on the stability of triangular points:

\begin{tabular}{lll}
\hline$\varepsilon$ & $\varepsilon^{\prime}$ & $\mu_{c}$ \\
\hline $\mathbf{0 . 0 0}$ & 0.00 & \\
$\mathbf{0 . 0 0}$ & 0.01 & $0.0385209=\mu_{0}$ \\
$\mathbf{0 . 0 0}$ & 0.06 & 0.0351322 \\
$\mathbf{0 . 0 0}$ & 0.11 & 0.0181890 \\
$\mathbf{0 . 0 0}$ & 0.16 & 0.00124583 \\
$\mathbf{0 . 0 0}$ & 0.21 & -0.0156974 \\
$\mathbf{0 . 0 0}$ & 0.26 & -0.0326406 \\
$\mathbf{0 . 0 0}$ & 0.31 & -0.0495838 \\
& & -0.0665270 \\
\hline
\end{tabular}

When there is no perturbation in the Centrifugal force i.e $\varepsilon^{\prime}=0, \mu_{c}=\mu_{0}$, and for $\varepsilon^{\prime}>0, \mu_{c}<\mu_{0}$ establishing that centrifugal force is a destabilizing force in the presence of heterogeneous factors, provided the Coriolis force is kept constant. 
Table 4: Effect of a perturbation in the Coriolis force with heterogeneous factors on the stability of triangular points:

\begin{tabular}{lll}
$\varepsilon$ & $\varepsilon^{\prime}$ & $\mu_{c}$ \\
\hline $\mathbf{0 . 0 0}$ & 0.00 & \\
$\mathbf{0 . 0 1}$ & 0.00 & $0.0385209=\mu_{0}$ \\
$\mathbf{0 . 0 6}$ & 0.00 & 0.0449414 \\
$\mathbf{0 . 1 1}$ & 0.00 & 0.0770443 \\
$\mathbf{0 . 1 6}$ & 0.00 & 0.1091470 \\
$\mathbf{0 . 2 1}$ & 0.00 & 0.1412500 \\
$\mathbf{0 . 2 6}$ & 0.00 & 0.1733530 \\
$\mathbf{0 . 3 1}$ & 0.00 & 0.2054560 \\
& & 0.2375590
\end{tabular}

When there is no perturbation in the Coriolis force, ie $\varepsilon=0, \mu_{c}=\mu_{0}$ for $\varepsilon>0, \mu_{c}>\mu_{0}$, establishing that Coriolis force is a stabilizing force in the presence of heterogeneous factors, provided the centrifugal force is kept constant.

Table 5: Effect of perturbations in the Coriolis and centrifugal forces with heterogeneous factors on the stability of the triangular points:

\begin{tabular}{lll}
\hline$\varepsilon$ & $\varepsilon^{\prime}$ & $\mu_{c}$ \\
\hline $\mathbf{0 . 0 0}$ & & \\
$\mathbf{0 . 0 1}$ & 0.00 & $0.03852090=\mu_{0}$ \\
$\mathbf{0 . 0 6}$ & 0.01 & 0.04155280 \\
$\mathbf{0 . 1 1}$ & 0.06 & 0.05671250 \\
$\mathbf{0 . 1 6}$ & 0.11 & 0.07187220 \\
$\mathbf{0 . 2 1}$ & 0.16 & 0.08703190 \\
$\mathbf{0 . 2 6}$ & 0.21 & 0.10219200 \\
$\mathbf{0 . 3 1}$ & 0.26 & 0.11735100 \\
& 0.31 & 0.13251100
\end{tabular}

For $\varepsilon=0$ and $\varepsilon^{\prime}=0, \mu_{c}=\mu_{0}$, and for $\varepsilon>0 \varepsilon^{\prime}>0, \mu_{c}>\mu_{0}$, hence perturbation in both parameters establish a stabilizing effect. 
Effect of a perturbation in the centrifugal force with heterogeneous factors on stability.

Table 6 STABILITY OF $L_{1}$

\begin{tabular}{cccccccc}
\hline $\boldsymbol{\beta}$ & $\boldsymbol{L}_{\mathbf{1}}$ & $\boldsymbol{\Omega}_{\boldsymbol{x} \boldsymbol{0}}^{\mathbf{0}}$ & $\boldsymbol{\Omega}_{\boldsymbol{y y}}^{\mathbf{0}}$ & $\boldsymbol{\Omega}_{\boldsymbol{x} \boldsymbol{0}}^{\mathbf{0}} \boldsymbol{\Omega}_{\boldsymbol{y}}^{\mathbf{0}}$ & $\boldsymbol{\lambda}_{\mathbf{1 , 2}}$ & $\boldsymbol{\lambda}_{\mathbf{3}, \mathbf{4}}$ & remark \\
\hline 1.0 & -1.23623 & 4.52992 & -0.764958 & -3.46520 & \pm 1.3220 & $\pm 1.4081 \mathrm{i}$ unstable \\
1.1 & -1.21074 & 5.08063 & -0.89031 & -4.52336 & \pm 1.4913 & $\pm 1.4261 \mathrm{i}$ & unstable \\
1.2 & -1.18834 & 5.64440 & -1.02220 & -5.76971 & \pm 1.6532 & $\pm 1.4529 \mathrm{i}$ & unstable \\
1.4 & -1.15065 & 6.80883 & -1.30442 & -8.88155 & \pm 1.9560 & $\pm 1.5236 \mathrm{i}$ & unstable \\
1.6 & -1.11994 & 8.01923 & -1.60962 & -12.9079 & \pm 2.2348 & $\pm 1.6077 \mathrm{i}$ unstable \\
1.8 & -1.09428 & 9.27229 & -1.93614 & -17.9525 & \pm 2.4943 & $\pm 1.6987 \mathrm{i}$ unstable \\
1.9 & -1.08292 & 9.91399 & -2.10700 & -20.8888 & \pm 2.6181 & $\pm 1.7457 \mathrm{i}$ unstable \\
\hline
\end{tabular}

Table 7 STABILITY OF $L_{2}$

\begin{tabular}{lccccccc}
\hline $\boldsymbol{\beta}$ & $\boldsymbol{L}_{2}$ & $\boldsymbol{\Omega}_{\boldsymbol{x} x}^{\mathbf{0}}$ & $\boldsymbol{\Omega}_{\boldsymbol{y y}}^{\mathbf{0}}$ & $\boldsymbol{\Omega}_{\boldsymbol{x} \boldsymbol{0}}^{\mathbf{0} \boldsymbol{Q}_{\boldsymbol{y y}}^{\mathbf{0}}}$ & $\boldsymbol{\lambda}_{\mathbf{1 , 2}}$ & $\boldsymbol{\lambda}_{\mathbf{3}, \mathbf{4}}$ & remark \\
\hline 1.0 & -0.168178 & 16.8008 & -6.90039 & -115.932 & \pm 3.7569 & $\pm 2.8660 \mathrm{i}$ & unstable \\
1.1 & -0.167182 & 16.8863 & -6.79316 & -114.712 & \pm 3.7659 & $\pm 2.8441 \mathrm{i}$ & unstable \\
1.2 & -0.166198 & 16.9728 & -6.6864 & -113.487 & \pm 3.7750 & $\pm 2.8220 \mathrm{i}$ & unstable \\
1.4 & -0.164261 & 17.1484 & -6.4742 & -111.022 & \pm 3.7934 & $\pm 2.7777 \mathrm{i}$ & unstable \\
1.6 & -0.162366 & 17.3274 & -6.2637 & -108.533 & \pm 3.8121 & $\pm 2.7329 \mathrm{i}$ & unstable \\
1.8 & -0.160512 & 17.5095 & -6.05474 & -106.915 & \pm 3.8311 & $\pm 2.6875 \mathrm{i}$ & unstable \\
1.9 & -0.159600 & 17.6017 & -5.95083 & -104.745 & \pm 3.1720 & $\pm 3.2265 \mathrm{i}$ & unstable \\
\hline
\end{tabular}


Table 8 STABILITY OF $L_{3}$

\begin{tabular}{lccccccc}
\hline $\boldsymbol{\beta}$ & $\boldsymbol{L}_{3}$ & $\boldsymbol{\Omega}_{\boldsymbol{x} x}^{\mathbf{0}}$ & $\boldsymbol{\Omega}_{\boldsymbol{y} \boldsymbol{0}}^{\mathbf{0}}$ & $\boldsymbol{\Omega}_{\boldsymbol{x} \boldsymbol{0}}^{\mathbf{0} \boldsymbol{\Omega}_{\boldsymbol{y}}^{\mathbf{0}}}$ & $\boldsymbol{\lambda}_{\mathbf{1 , 2}}$ & $\boldsymbol{\lambda}_{\mathbf{3}, \mathbf{4}}$ & remark \\
\hline 1.0 & 0.806425 & 17.3774 & -7.18869 & -124.921 & \pm 3.8330 & $\pm 2.9160 \mathrm{i}$ & unstable \\
1.1 & 0.748024 & 26.505 & -11.6025 & -307.523 & \pm 4.8800 & $\pm 3.5934 \mathrm{i}$ & unstable \\
1.2 & 0.698684 & 40.2683 & -18.3341 & -738.281 & \pm 6.1302 & $\pm 4.4323 \mathrm{i}$ & unstable \\
1.4 & 0.619602 & 93.3415 & -44.5705 & -4160.28 & \pm 9.5215 & $\pm 6.7741 \mathrm{i}$ & unstable \\
1.6 & 0.558745 & 223.884 & -109.541 & -24524.4 & \pm 14.8731 & $\pm 10.5293 \mathrm{i}$ & unstable \\
1.8 & 0.510383 & 578.914 & -286.75 & -166004 & \pm 24.0051 & $\pm 16.9729 \mathrm{i}$ & unstable \\
1.9 & 0.489858 & 972.263 & -483.266 & -469861 & \pm 31.1382 & $\pm 22.0135 \mathrm{i}$ & unstable \\
\hline
\end{tabular}

Effect of a perturbation in the Coriolis and centrifugal force with heterogeneous factors on stability

Table 9 stability of $L_{1}$

\begin{tabular}{lcccccccc}
\hline $\boldsymbol{\alpha}$ & $\boldsymbol{\beta}$ & $\boldsymbol{L}_{\mathbf{1}}$ & $\boldsymbol{\Omega}_{\boldsymbol{x} \boldsymbol{0}}^{\mathbf{0}}$ & $\boldsymbol{\Omega}_{\boldsymbol{y} \boldsymbol{0}}^{\mathbf{0}}$ & $\boldsymbol{\Omega}_{\boldsymbol{x} \boldsymbol{0}}^{\mathbf{0}} \boldsymbol{\Omega}_{\boldsymbol{y}}^{\mathbf{0}}$ & $\boldsymbol{\lambda}_{\mathbf{1 , 2}}$ & $\boldsymbol{\lambda}_{\mathbf{3} \mathbf{4}}$ & remark \\
\hline 1.0 & 1.0 & -1.23623 & 4.52992 & -0.764958 & -3.4652 & \pm 1.3220 & $\pm 1.4081 \mathrm{i}$ & unstable \\
1.1 & 1.1 & -1.21074 & 5.08063 & -0.890314 & -4.52336 & \pm 1.4229 & $\pm 1.4947 \mathrm{i}$ & unstable \\
1.2 & 1.2 & -1.18834 & 5.6444 & -1.02220 & -5.76971 & \pm 1.5214 & $\pm 1.5788 \mathrm{i}$ & unstable \\
1.4 & 1.4 & -1.15065 & 6.80883 & -1.30442 & -8.88155 & \pm 1.7125 & $\pm 1.7402 \mathrm{i}$ & unstable \\
1.6 & 1.6 & -1.11994 & 8.01923 & -1.60962 & -12.9079 & \pm 1.8967 & $\pm 1.8942 \mathrm{i}$ & unstable \\
1.8 & 1.8 & -1.09428 & 9.27229 & -1.93614 & -17.9525 & \pm 2.0750 & $\pm 2.0419 \mathrm{i}$ & unstable \\
1.9 & 1.9 & -1.08292 & 9.91399 & -2.10700 & -20.8888 & \pm 2.1622 & $\pm 2.1138 \mathrm{i}$ & unstable \\
\hline
\end{tabular}


Table 10 STABILITY OF $L_{2}$

\begin{tabular}{lcccccccc}
\hline $\boldsymbol{\alpha}$ & $\boldsymbol{\beta}$ & $\boldsymbol{L}_{\mathbf{2}}$ & $\boldsymbol{\Omega}_{\boldsymbol{x} x}^{\mathbf{0}}$ & $\boldsymbol{\Omega}_{\boldsymbol{y y}}^{\mathbf{0}}$ & $\boldsymbol{\Omega}_{\boldsymbol{x} \boldsymbol{0}}^{\mathbf{0}} \boldsymbol{\Omega}_{\boldsymbol{y y}}^{\mathbf{0}}$ & $\boldsymbol{\lambda}_{\mathbf{1 , 2}}$ & $\boldsymbol{\lambda}_{\mathbf{3 , 4}}$ & remark \\
\hline 1.0 & 1.0 & -0.168178 & 16.8008 & -6.9004 & -115.932 & \pm 3.7569 & $\pm 2.8660 \mathrm{i}$ & unstable \\
1.1 & 1.1 & -0.16718 & 16.8863 & -6.7932 & -114.712 & \pm 3.7321 & $\pm 2.8698 \mathrm{i}$ & unstable \\
1.2 & 1.2 & -0.16620 & 16.9728 & -6.6864 & -113.487 & \pm 3.7073 & $\pm 2.8736 \mathrm{i}$ & unstable \\
1.4 & 1.4 & -0.16426 & 17.1484 & -6.4742 & -111.022 & \pm 3.6572 & $\pm 2.8811 \mathrm{i}$ & unstable \\
1.6 & 1.6 & -0.16237 & 17.3274 & -6.2638 & -108.533 & \pm 3.6066 & $\pm 2.8886 \mathrm{i}$ & unstable \\
1.8 & 1.8 & -0.16051 & 17.5095 & -6.0547 & -106.015 & \pm 3.5555 & $\pm 2.8959 \mathrm{i}$ & unstable \\
1.9 & 1.9 & -0.15960 & 17.6017 & -5.9508 & -104.745 & \pm 3.5296 & $\pm 2.8996 \mathrm{i}$ & unstable
\end{tabular}

Table 11 STABILITY OF $L_{3}$

\begin{tabular}{ccccccccc}
\hline $\boldsymbol{\alpha}$ & $\boldsymbol{\beta}$ & $\boldsymbol{L}_{\mathbf{3}}$ & $\boldsymbol{\Omega}_{\boldsymbol{x} \boldsymbol{0}}^{\mathbf{0}}$ & $\boldsymbol{\Omega}_{\boldsymbol{y y}}^{\mathbf{0}}$ & $\boldsymbol{\Omega}_{\boldsymbol{x} \boldsymbol{0}}^{\mathbf{0}} \boldsymbol{\Omega}_{\boldsymbol{y y}}^{\mathbf{0}}$ & $\boldsymbol{\lambda}_{\mathbf{1 , 2}}$ & $\boldsymbol{\lambda}_{\mathbf{3}, \mathbf{4}}$ & remark \\
\hline 1.0 & 1.0 & 0.806425 & 17.377 & -7.18869 & -124.921 & \pm 3.8330 & $\pm 2.9160 \mathrm{i}$ & unstable \\
1.1 & 1.1 & 0.748024 & 26.505 & -11.6025 & -307.523 & \pm 4.8536 & $\pm 3.6131 \mathrm{i}$ & unstable \\
1.2 & 1.2 & 0.698684 & 40.2683 & -18.3341 & -738.281 & \pm 6.0874 & $\pm 4.4635 \mathrm{i}$ & unstable \\
1.4 & 1.4 & 0.619602 & 93.3415 & -44.5705 & -4160.28 & \pm 9.4658 & $\pm 6.8140 \mathrm{i}$ & unstable \\
1.6 & 1.6 & 0.558745 & 223.884 & -109.541 & -24524.4 & \pm 14.8194 & $\pm 10.5674 \mathrm{i}$ & unstable \\
1.8 & 1.8 & 0.510383 & 578.914 & -286.75 & -166004 & \pm 23.9606 & $\pm 17.0044 \mathrm{i}$ & unstable \\
1.9 & 1.9 & 0.489858 & 972.263 & -483.266 & -469861 & \pm 31.0998 & $\pm 22.0408 \mathrm{i}$ & unstable \\
\hline
\end{tabular}

Because $\Omega_{x x}^{0} \Omega_{y y}^{0}<0$, the discriminant is positive and the four roots of the characteristic equation computed as $\lambda_{1}=s, \lambda_{2}=$ $-s, \lambda_{3}=i t, \lambda_{4}=-i t$

\section{Discussion}

Equations (1)-(4) describe the motion of a test particle in the circular R3BP when small perturbations in the Coriolis and centrifugal forces are given and the primaries of the system are heterogeneous spheroids with three layers having distinct densities. In this 
problem, if the effects due to the heterogeneity of the primaries are neglected, then $n^{2}=1$, and Eqs (1)-(4) agree with those of Bhatnagar and Hallan (1978).

Eqs.20 and 21, table1 and figure1, represent the coordinates of the triangular points. The positions of triangular points are affected by a small perturbation of the centrifugal force and the heterogeneity of both primaries. We observe that with perturbations and heterogeneity, triangular points no longer form equilateral triangles with the primaries as they do in the classical case but scalene triangles, and they move closer to the line joining the primaries.

Eqs.22 to25, table 2 and figure2, show the location of the collinear points which are affected by a small perturbation of the centrifugal force and heterogeneous factors of the primaries. In the presence of all perturbation, $L_{1}$ is shifted towards the smaller primary. $L_{2}$ moves towards the bigger primary while $L_{3}$ also moves towards the bigger primary.

Eq.32 to 35, tables 3-5, give the critical value of the mass parameter of the system which depends upon small perturbations $\varepsilon$, $\varepsilon$ 'in the Coriolis and centrifugal forces and heterogeneous factors $k_{i}$. We observe that the centrifugal force and the heterogeneous factors all have destabilizing effects, and therefore the size of the region of stability decreases with the increase in the value of these parameters, while the Coriolis force has a stabilizing effect on triangular points.

From Eqs.36 to 40 and tables 6 to 11, we see that heterogeneous factors and perturbations in Coriolis and centrifugal forces are unable to change the instability character of the collinear points. Hence the collinear Libration points remain unstable under these perturbations.

\section{Conclusion}

By considering both primaries as heterogeneous spheroids under the influence of small perturbations in the Coriolis and centrifugal forces we have determined the positions of equilibrium points and have examined their linear stability. It is found that their positions are affected by a small change in the centrifugal force and heterogeneous factors of the primaries. We further observed that collinear

points remain unstable and triangular points are stable for $0<\mu<\frac{1}{2}$ and unstable for $\mu_{c} \leq \mu \leq \frac{1}{2}$ where $\mu_{c}$ is dependent on small perturbation in the centrifugal force and heterogeneous factors, all of which have destabilizing tendencies resulting in a decrease in the region of stability, except for the Coriolis force.

www.scirj.org

(C) 2020, Scientific Research Journal

http://dx.doi.org/10.31364/SCIRJ/v8.i11.2020.P1120821

This publication is licensed under Creative Commons Attribution CC BY. 


\section{References}

[1] Witner, A. (1941), The analytical foundations of celestial mechanics, p. 372, Princeton Yamada, K., Princeton

[2] Szebehely, V. (1967) Theory of Orbits: The Restricted Problem of Three bodies. Academic Press, New York

[3] Chernikov, Yu. A.(1970): The photogravitational restricted three body problem. Soviat Astron. A.J.14,I

[4] Sharma R.K, (1982) Sun and Planetary System, W Frieckey \& G. Teleky (eds), 435-436 by D Riedel.435

[5] Simmons J.F.L, McDonald, A.J.C.: The restricted 3-body problem with radiation pressure. Celestial Mechanics.35, 145-187

[6] Ragos O., Zagouras C.: Periodic solutions about the out of plane equilibrium points in the photogravitational restricted three-body problem. Celestial Mechanics.44(1-2),135-154 (1988).

[7] El-Shaboury S.M.:(1991). Equilibrium solutions of the restricted problem of $2 \times 2$ axisymmetric rigid bodies, Celestial Mechanics and Dynamics. Astro 50, 199-208

[8] Khanna,U., Bhatnagar,K.B. (1999),Existence and Stability of Libration points in the restricted three-body problem when the smaller primary is a triaxial rigid body and the bigger one an oblate spheroid. Indian J Pure.Appl.math.30(7),721-733

[9] Idrisi, M.J., Taqvi, Z.A.(2013),: Restricted three-body problem when one of the primaries is an ellipsoid. Astrophys. Space Sci. 348,41

[10] Singh,J., Taura, J.J.,(2013): Motion in the restricted three body problem.Astrophysics.Space Sci. 343,95-106

[11] Suraj Md, M.R Hassan,Md Chand Asique ,(2014),: The Photo-Gravitational restricted three body problem when the primaries are heterogeneous spheroid with three layers. J of Astronaut Sci.61,133-155

[12] Singh,J.,Bello.(2014); Effect of perturbations in the Coriolis and Centrifugal Forces on the Stability of $L_{4}$ in the Relativistic R3BP J of Astrophys. 35, 701-713

[13] Singh,J.,Taura,J.J.(2015): Collinear Libration points in the Photogravitational CR3BP with Zonal Harmonics and Potential From a belt. Astronomy and Astrophysics, 5, 155-165

www.scirj.org

(C) 2020, Scientific Research Journal

http://dx.doi.org/10.31364/SCIRJ/v8.i11.2020.P1120821

This publication is licensed under Creative Commons Attribution CC BY. 